\title{
Cardiovascular Events in Moderately to Severely Obese Obstructive Sleep Apnea Patients on Positive Airway Pressure Therapy
}

\author{
Anna Maria Marotta ${ }^{a}$ Jean-Christian Borel ${ }^{b}$ Louis M. Galerneau ${ }^{c}$ \\ Renaud Tamisier ${ }^{b, c}$ Maria R. Bonsignore ${ }^{a}$ Jean Louis Pépin ${ }^{b, c}$ \\ ${ }^{a}$ Dipartimento Biomedico di Medicina Interna e Specialistica, University of Palermo, Palermo, Italy; \\ b Université Grenoble Alpes, HP2, Inserm, U1042, and ' ${ }^{\mathrm{C} H U}$ de Grenoble, Laboratoire EFCR, Pôle Thorax et \\ Vaisseaux, Grenoble, France
}

\section{Keywords}

Sleep-disordered breathing · Longitudinal studies ·

Continuous positive airway pressure $\cdot$ Noninvasive

ventilation · Prognosis

\begin{abstract}
Background: In moderately to severely obese patients with obstructive sleep apnea (OSA), the effects of long-term positive airway pressure (PAP) treatment on cardiovascular risk are poorly defined. Purpose: To assess the effect of continuous positive airway pressure (CPAP) or noninvasive ventilation (NIV) on the occurrence of cardiovascular events in obese OSA patients. Methods: We performed a noninterventional observational study in obese OSA patients recruited between 2007 and 2010 at the Sleep Center, University of Grenoble, treated with CPAP or NIV, and followed for 5.6 years by a single home care provider. Baseline clinical characteristics, blood chemistry, and respiratory and vascular function were assessed. Incident cardiovascular events were investigated by phone interviews. Results: A total of 103 patients (55 men, 48 women; age and body mass index [BMI] at diagnosis $54.1 \pm 10.5$ years and $40.3 \pm 5.5$, respectively [mean \pm standard deviation]; CPAP: $n=75$; NIV: $n=28$ ) agreed to participate in the study. Grade I, II, and III obesity occurred in
\end{abstract}

๑) 2017 S. Karger AG, Basel
$17.5,33.0$, and $49.5 \%$ of the sample, respectively. In patients using PAP treatment ( $n=69$ ), the mean nightly use was $6.3 \pm 2.4 \mathrm{~h}$. Thirty-one patients stopped PAP treatment during follow-up. Three patients on NIV died. Nonfatal cardiovascular events ( $n=27$ ) occurred in 19 patients, who were older and showed higher number of comorbidities and triglyceride levels than patients without events. In the patients who interrupted treatment, the event rate was high and increased with the number of comorbidities, while BMI at baseline did not predict events. Conclusions: The study suggests that regular PAP treatment may be associated with protection against cardiovascular risk in obese OSA patients, especially in the presence of multiple comorbidities.

(c) 2017 S. Karger AG, Basel

\section{Introduction}

Obesity has reached epidemic proportions, with a high prevalence of class III obesity, i.e., a body mass index $(\mathrm{BMI})>40$ [1]. Studies consistently show an association

A.M. Marotta and J.-C. Borel are co-first authors. M.R. Bonsignore and J.L. Pépin are co-senior authors. 


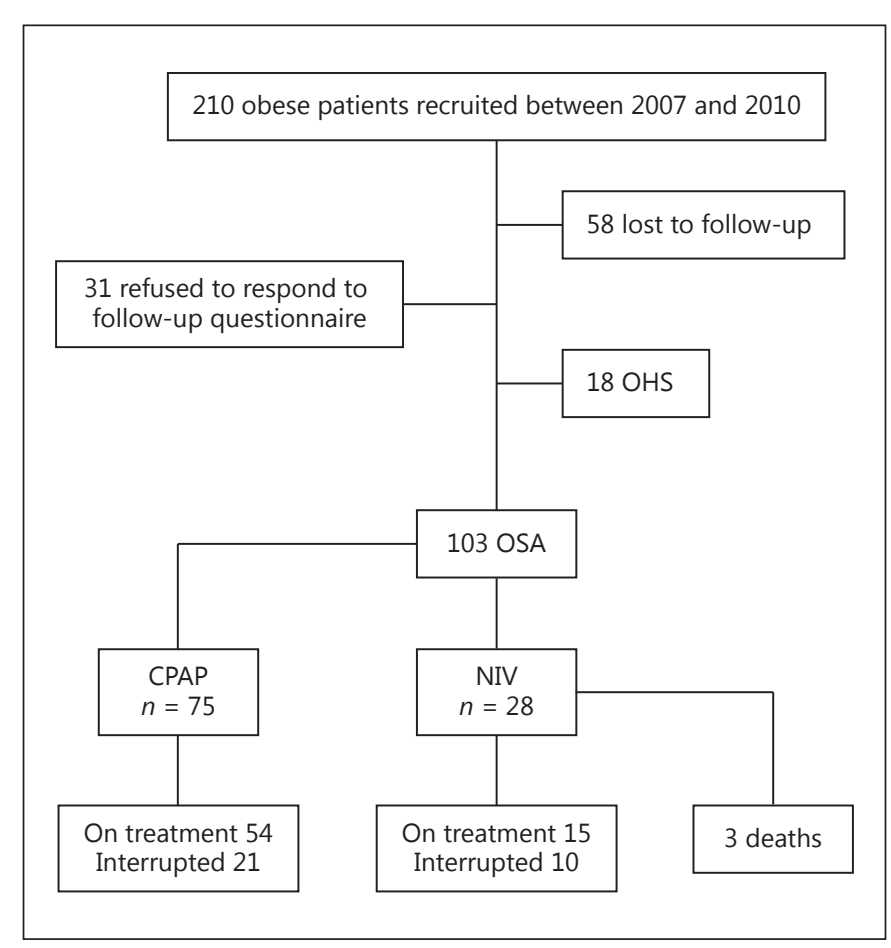

Fig. 1. Study flowchart. CPAP, continuous positive airway pressure; NIV, noninvasive ventilation; OHS, obesity hypoventilation syndrome; OSA, obstructive sleep apnea.

between obesity and increased mortality, mainly from heart disease, cancer, and diabetes [2-4].

Obstructive sleep apnea (OSA) is highly prevalent in patients with severe obesity $[5,6]$ and could contribute to increased cardiovascular risk [7]. In a prospective cohort of overweight to mildly obese OSA patients (mean BMI: $30.7 \pm 4.4$ ), increased rates of fatal and nonfatal cardiovascular events were documented in severe untreated OSA, while treatment with continuous positive airway pressure (CPAP) was protective [8]. However, other cohort studies underlined the fact that a high incidence of cardiovascular disease persists even in CPAP-treated OSA patients [9]. Comorbidities appear as the main contributors to mortality in these patients $[10,11]$, suggesting that the high cardiovascular risk might be linked more to obesity or other risk factors than to OSA. Accordingly, Salord et al. [12] did not find any difference in cardiometabolic profile or prevalence of metabolic syndrome between morbidly obese patients with or without OSA, suggesting a "ceiling effect" of extreme obesity. Recently, Kendzerska et al. [13] reported that obesity and nocturnal oxygen desaturation were associated with a high incidence of cardiovascular events in OSA patients.
Randomized controlled trials in morbidly obese patients with OSA or obesity hypoventilation syndrome (OHS) suggest that treatment of sleep-disordered breathing has no impact on inflammatory [14] or metabolic markers $[15,16]$ or on arterial stiffness assessed by pulse wave velocity (PWV) [17]. However, so far only few longterm studies have assessed cardiovascular morbidity and mortality in severely obese patients treated by positive airway pressure (PAP) therapies [18-21].

The primary aim of this study was to collect data on mortality and incident cardiovascular events in a noninterventional observational study over a period of 5 years in obese patients with OSA treated with PAP therapies and followed by a single home care provider. The secondary goal was to identify whether baseline comorbidities, medications, and existing vascular remodeling (assessed by arterial stiffness and endothelial function measurements at baseline) could predict cardiovascular events/ death during follow-up.

\section{Patients and Methods}

Study Design

This is a noninterventional, observational study from a prospective cohort.

\section{Patients}

Between 2007 and 2010, a cohort of 210 patients with obesity and OSA or OHS referred to the Sleep Center, University of Grenoble, was constituted. Patients with cognitive impairment and other causes of alveolar hypoventilation, i.e., neuromuscular disease or chest wall disorders, were not included. At inclusion, all patients provided written informed consent, and the study protocol was approved by the institutional review board (IRB-6705). In the present analysis, patients with OHS were excluded (see Results and Fig. 1).

\section{Follow-Up}

Out of the original cohort, 152 patients on CPAP or noninvasive ventilation (NIV) were followed regularly, i.e., every 4-6 months, by the same nonprofit home care provider (AGIR à dom). Data on objective compliance to CPAP/NIV were regularly recorded by AGIR à dom and are reported as mean nightly use (h). This was the guarantee for a homogeneous care pathway and follow-up. The choice of ventilatory treatment modality was based on clinical variables, and NIV was prescribed instead of CPAP in cases of intolerance to high CPAP level, incomplete resolution of nocturnal respiratory events on CPAP, or lack of normalization of bicarbonate levels.

A standardized follow-up questionnaire (online suppl. Appendix, see www.karger.com/doi/10.1159/000454988) including data on PAP therapy compliance, smoking status, weight evolution, and if applicable alternative treatment, i.e., mandibular advancement device or bariatric surgery, was administered. 
Information on hospital admission for cerebrovascular events, myocardial infarction, unstable angina, stroke, arrhythmia, or acute coronary events that needed coronary bypass surgery or angioplasty during the follow-up period was collected. When a patient reported an event on the questionnaire, this event was confirmed by checking hospital medical chart or by calling the patient's general practitioner.

\section{Baseline Assessment of the Patients Involved in the Cohort}

Baseline assessment included medical history (smoking status, comorbidities, and medications), anthropometric measurements (weight, height, neck, waist, and hip circumferences, smoking status, office arterial systolic and diastolic blood pressure [22], pulmonary function tests, polysomnography, biological parameters for metabolic and inflammatory profile, arterial stiffness (assessed by $\mathrm{PWV}$ ), and endothelial function (assessed by reactive hyperemia-peripheral arterial tonometry [RH-PAT]).

Pulmonary Function Tests and Blood Gases. An arterial blood sample was drawn during wakefulness to measure blood gases. Spirometry and plethysmography were performed (body plethysmograph Bodybox 5500, Medisoft) according to the recommendations of the European Respiratory Society [23].

Sleep Study and Sleepiness Assessment. Overnight full polysomnography (Natus Technology, DeltaMed SA) under baseline conditions was recorded to characterize abnormal respiratory events during sleep according to standard criteria [24]. Subjective sleepiness was assessed by the Epworth Sleepiness Scale [25].

Biomarkers. At waking up after a 10-h fast, a venous blood sample was drawn to measure serum fasting glucose, triglycerides, and cholesterol according to standard procedures (Modular 700; Roche, Meylan, France). Glycated hemoglobin A1c was measured by ion-exchange high-performance liquid chromatography with double-wavelength detection (Variant II ${ }^{\circledR}$; Bio-Rad, Hercules, CA, USA). Serum insulin was measured using a radioimmunometric sandwich assay (Cisbio Int., Gif-Sur-Yvette, France), and insulin resistance was estimated by the homeostasis model assessment index [26]. Occurrence of metabolic syndrome was assessed according to the NCEP-ATPIII criteria [27].

The serum high-sensitivity C-reactive protein level was measured using automated immunonephelometry (Behring Nephelometer II Analyzer; Dade Behring, Germany). Cytokines tumor necrosis factor- $\alpha$ was measured by enzyme immunoassay (Immunotech SAS, Marseille, France). Leptin and interleukin 6 were measured by radioimmunoassay (LINCO Research, USA).

Arterial Stiffness and Vascular Endothelial Function. Carotidfemoral PWV was measured as a marker of arterial stiffness at baseline [28]. To determine PWV, 2 pulse transducers were fixed on the skin over the right common carotid and femoral arteries [26]. The time delay was measured with a Complior device (Alam Medical, Vincennes, France) between the feet of simultaneously recorded pulse waves and averaged over 10 consecutive cycles. The carotid-femoral PWV was calculated as the distance between the arterial sites divided by the time delay. The measured PWV values were compared to those recorded in a reference population according to age and blood pressure category [29]. Vascular endothelial function was assessed during wakefulness as pulse volume amplitude (PVA) under baseline conditions and during RH (RHPAT, Endo-PAT device; Itamar Medical Ltd.) [30, 31]. PVA was measured with the subjects placed in the supine position, in a quiet, temperature-controlled set. The PVA apparatus consist of a fin- ger-mounted probe that surrounds the fingertip with an electronically controlled, inflatable, pressurized air cushion confined within a rigid external case. The pressure changes within the probe that accompany PVA changes in the fingertip are transmitted to a personal computer where the signal is bandpass filtered $(0.3-30 \mathrm{~Hz})$, amplified, displayed, and stored. PVA was analyzed at rest and during $\mathrm{RH}$ elicited by the release of an upper arm blood pressure cuff inflated above systolic pressure for 5 min. Digital PVA-RH was calculated as the ratio of the average PVA over a 1-min interval starting $1 \mathrm{~min}$ after cuff deflation $(\mathrm{RH})$ divided by the average PVA measured for $1 \mathrm{~min}$ before cuff inflation (baseline). The PVA from the index finger of the other, nonischemic hand (which was not subject to $\mathrm{RH}$ ) was measured throughout the study to assess any drift in the magnitude of the signal due to systemic factors.

\section{Statistical Analysis}

Data are reported as mean \pm standard deviation for continuous variables, and as frequencies for nominal variables. The unpaired $t$ test was used to compare variables between patients accepting and refusing to respond to the questionnaire and between OSA patients treated with CPAP or NIV; log transformation or MannWhitney $\mathrm{U}$ test was applied to nonnormally distributed variables before statistical analysis. Frequencies were compared by the $\chi^{2}$ test. Simple linear regression was used to analyze relationships between continuous variables. Multivariate stepwise linear regression was used to analyze correlates of PWV or RH-PAT. Two-way analysis of variance was used to compare cardiovascular event rates according to continuing or interrupted PAP treatment, and number of comorbidities. Statistical significance was assumed at $p<0.05$ for all tests applied in this study. The Statview 5.0 for Windows software was used for all analyses.

\section{Results}

Figure 1 shows the study flowchart. Out of 210 patients, 58 were lost to follow-up, and 31 refused to respond to the follow-up questionnaire; they were comparable for age, gender distribution, BMI, and severity of OSA to the patients who accepted to participate in the longitudinal analysis (data not shown). However, the patients who refused the questionnaire were all nonsmokers at inclusion, compared to $18.5 \%$ of current smokers at baseline among those who responded $(p=0.013)$, and showed an almost double rate of cessation of PAP therapies during follow-up ( 46.7 vs. $25.5 \%, p=0.02)$. The OHS group ( $n=18$ ) was small, and all OHS patients were excluded from analysis. Four patients ( 1 man and 3 women) underwent uvulopalatopharyngoplasty before entering the study.

Table 1 reports the anthropometric characteristics and medical history at baseline of the 103 included OSA patients according to the treatment prescribed (CPAP or NIV). Gender distribution was similar in both groups. The mean BMI in the cohort was $40.3 \pm 5.5$; grade I, II, and III 
Table 1. Baseline anthropometrics, comorbidities, arterial blood gases, and sleep variables in obstructive sleep apnea patients according to treatment

\begin{tabular}{|c|c|c|c|c|}
\hline & All & CPAP & NIV & $p$ \\
\hline Men/women & $55 / 48$ & $41 / 34$ & $14 / 14$ & 0.67 \\
\hline Age, years & $54.1 \pm 10.5$ & $53.8 \pm 10.7$ & $55.1 \pm 10.3$ & 0.56 \\
\hline BMI & $40.3 \pm 5.5$ & $39.9 \pm 5.0$ & $41.4 \pm 6.6$ & 0.23 \\
\hline Neck circumference, $\mathrm{cm}$ & $43.6 \pm 5.1$ & $42.8 \pm 5.1$ & $45.3 \pm 6.4$ & 0.04 \\
\hline Waist to hip ratio & $0.98 \pm 0.13$ & $0.98 \pm 0.12$ & $0.98 \pm 0.14$ & 0.98 \\
\hline $\mathrm{SBP}, \mathrm{mm} \mathrm{Hg}$ & $130.6 \pm 13.9$ & $130.8 \pm 14.1$ & $130.1 \pm 13.6$ & 0.81 \\
\hline $\mathrm{DBP}, \mathrm{mm} \mathrm{Hg}$ & $74.4 \pm 12.0$ & $75.2 \pm 12.4$ & $72.3 \pm 10.7$ & 0.31 \\
\hline \multicolumn{5}{|l|}{ Comorbidities } \\
\hline Hypertension & $61.6 \%$ & $60.3 \%$ & $65.4 \%$ & 0.65 \\
\hline Myocardial infarction & $4 \%$ & $1.4 \%$ & $11.5 \%$ & 0.02 \\
\hline Type 2 diabetes & $24.5 \%$ & $20.5 \%$ & $34.6 \%$ & 0.15 \\
\hline Stroke/transient ischemic attack & $1 \%$ & $1.4 \%$ & $0 \%$ & 0.55 \\
\hline High cholesterol & $21.6 \%$ & $19.2 \%$ & $26.9 \%$ & 0.41 \\
\hline Kidney failure & $1 \%$ & $1.4 \%$ & $0 \%$ & 0.55 \\
\hline Current smoking & $13.6 \%$ & $13.3 \%$ & $14.3 \%$ & 0.98 \\
\hline Previous smoking & $49.5 \%$ & $48.0 \%$ & $53.6 \%$ & 0.76 \\
\hline Respiratory disease & $17.2 \%$ & $16.4 \%$ & $19.2 \%$ & 0.75 \\
\hline Mean comorbidity number/patient & $1.33 \pm 0.99$ & $1.23 \pm 0.99$ & $1.62 \pm 0.90$ & 0.09 \\
\hline \multicolumn{5}{|l|}{ Arterial blood gases } \\
\hline $\mathrm{pH}$ & $7.42 \pm 0.02$ & $7.42 \pm 0.02$ & $7.42 \pm 0.02$ & 0.56 \\
\hline $\mathrm{PaCO}_{2}, \mathrm{kPa}$ & $5.25 \pm 0.37$ & $5.17 \pm 0.36$ & $5.48 \pm 0.33$ & 0.0001 \\
\hline $\mathrm{PaO}_{2}, \mathrm{kPa}$ & $10.4 \pm 1.12$ & $10.41 \pm 1.20$ & $10.39 \pm 1.18$ & 0.93 \\
\hline $\mathrm{HCO}^{3-}, \mathrm{mmol}$ & $25.2 \pm 1.7$ & $24.8 \pm 1.6$ & $26.1 \pm 1.6$ & 0.0003 \\
\hline \multicolumn{5}{|l|}{ Sleep study } \\
\hline AHI, $n / \mathrm{h}$ & $47.0 \pm 32.5$ & $50.3 \pm 33.0$ & $38.0 \pm 29.8$ & 0.10 \\
\hline Lowest $\mathrm{SaO}_{2}, \%$ & $73.3 \pm 11.1$ & $74.0 \pm 9.6$ & $71.5 \pm 14.5$ & 0.32 \\
\hline Mean $\mathrm{SaO}_{2}, \%$ & $90.9 \pm 4.0$ & $90.9 \pm 4.2$ & $90.9 \pm 3.6$ & 0.99 \\
\hline $\mathrm{CT}<90 \%$ & $23.2 \pm 24.4$ & $23.0 \pm 23.7$ & $23.8 \pm 26.7$ & 0.88 \\
\hline ESS & $11.5 \pm 5.1$ & $10.5 \pm 5.2$ & $14.1 \pm 3.8$ & 0.001 \\
\hline
\end{tabular}

Continuous variables are reported as means \pm standard deviation, frequencies as percentage. Bold values indicate statistical significance $(\mathrm{p}<0.05)$. AHI, apnea-hypopnea index; BMI, body mass index; CPAP, continuous positive airway pressure; $\mathrm{CT}<90 \%$, cumulative time spent at an $\mathrm{SaO}_{2}<90 \%$; DBP, diastolic blood pressure; ESS, Epworth Sleepiness Scale; NIV, noninvasive ventilation; SBP, systolic blood pressure.

obesity occurred in $17.5,33.0$, and $49.5 \%$ of the sample, respectively, without differences between the CPAP and the NIV group (data not shown). Compared to the CPAP group, patients on NIV showed a trend towards higher $\mathrm{BMI}$ and significantly higher neck circumference, $\mathrm{PaCO}_{2}$ and bicarbonate levels. Nine out of 28 patients on NIV (32\%) had bicarbonate levels $>27 \mathrm{~mm} / \mathrm{L}$, compared to 6 out of 75 patients on CPAP $(8 \%, p=0.002)$. The prevalence of comorbidities was similar in the 2 treatment groups, with the exception of previous myocardial infarction, which was more frequent in NIV-treated compared to CPAP-treated patients. The mean number of comorbidities per patient tended to be higher in the NIV group $(p=0.09)$. Spirometry was mostly normal and similar in the 2 groups (data not shown). The sleep study data showed a trend for a higher apnea-hypopnea index (AHI) in the CPAP group, with no differences in lowest or mean $\mathrm{SaO}_{2}$, or cumulative time spent at an $\mathrm{SaO}_{2}<90 \%$ between groups. NIV-treated patients showed more severe subjective daytime sleepiness than CPAP-treated patients.

Metabolic variables such as fasting glucose, serum insulin, lipid profile, and prevalence of metabolic syndrome were similar in the CPAP and the NIV group (Table 2). Serum tumor necrosis factor- $\alpha$ values at baseline, available in 39 patients (29 on CPAP, 10 on NIV), were significantly higher in NIV-treated patients.

Regarding arterial stiffness, most patients showed higher than predicted PWV values at baseline $(p<0.0001$ 
Table 2. Metabolic variables in obstructive sleep apnea patients according to treatment

\begin{tabular}{lllll}
\hline & All & CPAP & NIV & $p$ \\
\hline Fasting blood glucose, $\mathrm{mmol} / \mathrm{L}$ & $6.55 \pm 2.61$ & $6.27 \pm 1.86$ & $7.25 \pm 3.86$ & 0.10 \\
Fasting insulin, $\mu \mathrm{U} / \mathrm{mL}$ & $14.3 \pm 18.5$ & $14.2 \pm 20.4$ & $14.7 \pm 12.6$ & 0.91 \\
HOMA-IR & $5.59 \pm 12.6$ & $5.61 \pm 13.9$ & $5.53 \pm 8.68$ & 0.48 \\
HbAlc, $\%$ & $6.3 \pm 1.2$ & $6.25 \pm 1.07$ & $6.61 \pm 1.60$ & 0.25 \\
Triglycerides, g/L & $1.51 \pm 0.73$ & $1.45 \pm 0.76$ & $1.69 \pm 0.59$ & 0.15 \\
Total cholesterol, g/L & $1.96 \pm 0.44$ & $1.95 \pm 0.45$ & $1.98 \pm 0.42$ & 0.76 \\
HDL cholesterol, g/L & $0.44 \pm 0.12$ & $0.43 \pm 0.12$ & $0.45 \pm 0.13$ & 0.46 \\
LDL cholesterol, g/L & $1.23 \pm 0.37$ & $1.23 \pm 0.38$ & $1.20 \pm 0.36$ & 0.71 \\
TNF- $\alpha, \mathrm{pg} / \mathrm{mL}(n=39)$ & $8.31 \pm 6.84$ & $6.97 \pm 6.11$ & $12.20 \pm 7.67$ & $\mathbf{0 . 0 3 5}$ \\
Leptin, ng/mL $(n=85)$ & $33.1 \pm 24.7$ & $32.0 \pm 22.1$ & $36.1 \pm 30.9$ & 0.50 \\
IL-6, pg/mL $(n=77)$ & $14.8 \pm 11.2$ & $14.2 \pm 11.4$ & $16.2 \pm 10.5$ & 0.48 \\
hsCRP, mg/L $(n=94)$ & $7.59 \pm 7.51$ & $7.74 \pm 7.83$ & $7.23 \pm 6.76$ & 0.77 \\
Metabolic syndrome & $82.5 \%$ & $80.0 \%$ & $89.3 \%$ & 0.27 \\
\hline
\end{tabular}

Continuous variables are reported as means \pm standard deviation, frequencies as percentage. The bold value indicates statistical significance $(\mathrm{p}<0.05)$. CPAP, continuous positive airway pressure; HbAlc, glycated hemoglobin A1c; HDL, high-density lipoprotein; HOMA-IR, homeostasis model assessment of insulin resistance; hsCRP, high-sensitivity C-reactive protein; IL-6, interleukin 6; LDL, low-density lipoprotein; NIV, noninvasive ventilation; TNF- $\alpha$, tumor necrosis factor- $\alpha$.

by paired $t$ test, Fig. 2), without differences between treatment groups (CPAP: $9.74 \pm 1.83 \mathrm{~m} / \mathrm{s}$; NIV: $9.38 \pm 2.19$ $\mathrm{m} / \mathrm{s} ; p=0.44)$. The response to $\mathrm{RH}$ was also similar in CPAP- and NIV-treated patients (RH-PAT ratio: CPAP $0.40 \pm 0.28$; NIV: $0.53 \pm 0.29 ; p=0.10$ ). In univariate linear regression, $\mathrm{PWV}$ at baseline correlated positively with age $\left(R^{2}=0.24, p<0.0009\right)$ and systolic blood pressure $\left(R^{2}=\right.$ $0.13, p<0.0001)$. Stepwise multiple regression confirmed the independent association of PWV with both age and systolic blood pressure $\left(R^{2}=0.34, p<0.0001\right)$, while BMI, AHI, or lowest $\mathrm{SaO}_{2}$ did not stay in the model.

\section{Longitudinal Analysis}

The mean follow-up of the cohort was $5.5 \pm 1.4$ years, without differences between the CPAP $(5.6 \pm 1.4$ years) and the NIV group $(5.4 \pm 1.3$ years). The mean BMI change during follow-up in the entire cohort was $-1.6 \pm$ 7.0; after exclusion of patients who underwent bariatric surgery $(n=13)$, no significant differences in BMI or BMI change were found between obese OSA patients with and without cardiovascular events during follow-up. The mean objective compliance to treatment was $6.3 \pm 2.5 \mathrm{~h} /$ night in the NIV group and $6.2 \pm 2.4 \mathrm{~h} /$ night in the CPAP group (not significant). During follow-up, 3 patients on NIV died (mean duration of their follow-up: $6.7 \pm 0.7$ years); the causes of death were cancer (1), cerebrovascular accident (1), and undetermined cause (1).

$\mathrm{CPAP} / \mathrm{NIV}$ in Obese OSA Patients at

High Cardiovascular Risk

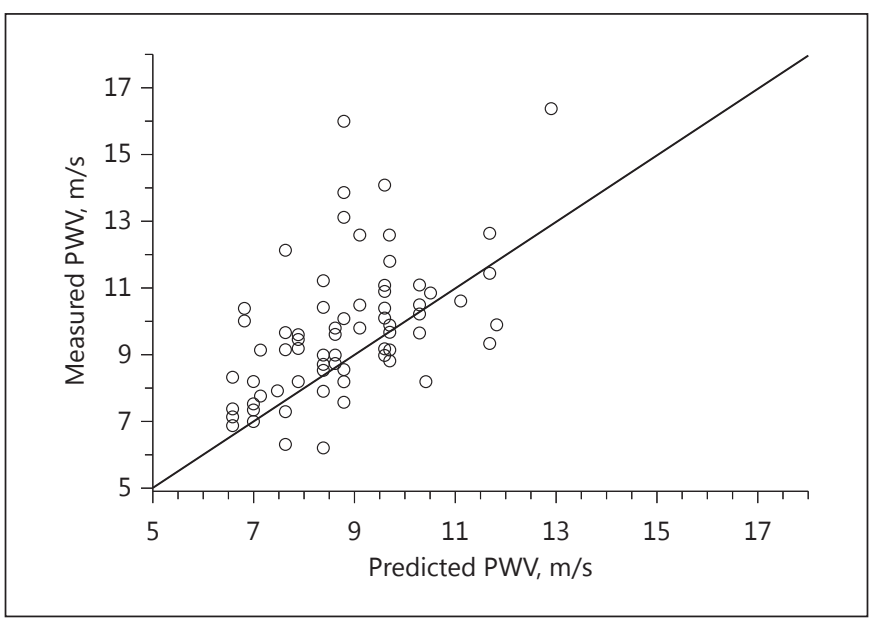

Fig. 2. Pulse wave velocity (PWV) at diagnosis was increased in most patients with obstructive sleep apnea. Predicted PWV was calculated according to the formula by Boutouyrie et al. [29].

Unique or multiple nonfatal cardiovascular events $(n=27)$ occurred in 19 patients: myocardial infarction (5 events), angina pectoris requiring hospitalization (3 events), coronary artery bypass surgery or angioplasty (5 events), aortic or carotid artery surgery (5 events), and cerebrovascular accidents (9 events). Compared to patients without events, patients who experienced events 
Table 3. Differences at baseline between obstructive sleep apnea patients with and without late cardiovascular events during follow-up

\begin{tabular}{|c|c|c|c|}
\hline & Without events & With events & $p$ \\
\hline Men/women & $42 / 39$ & $13 / 9$ & 0.55 \\
\hline Age, years & $52.9 \pm 10.2$ & $58.7 \pm 10.7$ & 0.02 \\
\hline $\mathrm{BMI}$ & $40.6 \pm 5.7$ & $39.1 \pm 4.8$ & 0.26 \\
\hline \multicolumn{4}{|l|}{ Comorbidities } \\
\hline Hypertension & $59 \%$ & $71 \%$ & 0.30 \\
\hline Myocardial infarction & $2.6 \%$ & $9.5 \%$ & 0.15 \\
\hline Type 2 diabetes & $23.1 \%$ & $28.6 \%$ & 0.60 \\
\hline Cerebrovascular accident & $1.3 \%$ & $0 \%$ & 0.60 \\
\hline High cholesterol & $17.9 \%$ & $33.3 \%$ & 0.13 \\
\hline Respiratory disease & $15.4 \%$ & $23.8 \%$ & 0.36 \\
\hline Kidney failure & $0 \%$ & $4.8 \%$ & 0.053 \\
\hline Mean number of comorbidities/patient & $1.22 \pm 0.92$ & $1.76 \pm 1.09$ & 0.02 \\
\hline \multicolumn{4}{|l|}{ Arterial blood gases } \\
\hline $\mathrm{pH}$ & $7.43 \pm 0.02$ & $7.42 \pm 0.02$ & 0.14 \\
\hline $\mathrm{PaCO}_{2}, \mathrm{kPa}$ & $5.22 \pm 0.35$ & $5.37 \pm 0.43$ & 0.11 \\
\hline $\mathrm{PaO}_{2}, \mathrm{kPa}$ & $10.43 \pm 1.20$ & $10.31 \pm 1.19$ & 0.68 \\
\hline $\mathrm{HCO}_{3}{ }^{-}, \mathrm{mmol}$ & $25.1 \pm 1.6$ & $25.5 \pm 1.8$ & 0.27 \\
\hline \multicolumn{4}{|l|}{ Sleep parameters } \\
\hline AHI, $n / \mathrm{h}$ & $48.8 \pm 34.5$ & $41.0 \pm 24.0$ & 0.33 \\
\hline Lowest $\mathrm{SaO}_{2}, \%$ & $72.9 \pm 11.3$ & $74.5 \pm 10.6$ & 0.56 \\
\hline $\mathrm{CT}<90 \%$ & $21.9 \pm 22.1$ & $27.5 \pm 31.2$ & 0.35 \\
\hline ESS & $11.4 \pm 5.3$ & $12.0 \pm 4.4$ & 0.59 \\
\hline \multicolumn{4}{|l|}{ Metabolic variables } \\
\hline HOMA-IR & $5.55 \pm 13.31$ & $5.74 \pm 9.92$ & 0.62 \\
\hline $\mathrm{HbA1c}, \%$ & $6.3 \pm 1.2$ & $6.5 \pm 1.5$ & 0.46 \\
\hline Triglycerides, g/L & $1.43 \pm 0.71$ & $1.83 \pm 0.71$ & 0.03 \\
\hline Total cholesterol, g/L & $1.92 \pm 0.44$ & $2.10 \pm 0.44$ & 0.12 \\
\hline HDL cholesterol, g/L & $0.44 \pm 0.13$ & $0.43 \pm 0.09$ & 0.89 \\
\hline Metabolic syndrome & $80 \%$ & $91 \%$ & 0.24 \\
\hline \multicolumn{4}{|l|}{ Inflammatory markers } \\
\hline TNF- $\alpha, \mathrm{pg} / \mathrm{mL}(n=33 / 6)$ & $7.79 \pm 6.79$ & $11.17 \pm 7.00$ & 0.27 \\
\hline Leptin, ng/mL $(n=67 / 18)$ & $31.2 \pm 24.1$ & $40.3 \pm 26.2$ & 0.17 \\
\hline IL-6, pg/mL $(n=61 / 16)$ & $14.5 \pm 11.5$ & $16.0 \pm 9.8$ & 0.63 \\
\hline hsCRP, mg/L $(n=75 / 19)$ & $7.66 \pm 7.90$ & $7.34 \pm 5.87$ & 0.87 \\
\hline \multicolumn{4}{|l|}{ Vascular function } \\
\hline $\operatorname{PWV}(n=68 / 18)$ & $9.50 \pm 1.93$ & $10.15 \pm 1.91$ & 0.20 \\
\hline RH-PAT ratio $(n=46 / 17)$ & $0.47 \pm 0.29$ & $0.37 \pm 0.26$ & 0.19 \\
\hline Frequency of RH-PAT $>0.4$ & $56 \%$ & $41 \%$ & 0.28 \\
\hline
\end{tabular}

Continuous variables are reported as means \pm standard deviation, frequencies as percentage. Bold values indicate statistical significance $(\mathrm{p}<0.05)$. AHI, apnea-hypopnea index; BMI, body mass index; CT <90\%, cumulative time spent at an $\mathrm{SaO}_{2}<90 \%$; ESS, Epworth Sleepiness Scale; HbA1c, glycated hemoglobin A1c; HDL, high-density lipoprotein; HOMA-IR, homeostasis model assessment of insulin resistance; hsCRP, high-sensitivity C-reactive protein; IL-6, interleukin 6; PWV, pulse wave velocity; RH-PAT, reactive hyperemia-peripheral arterial tonometry; TNF- $\alpha$, tumor necrosis factor- $\alpha$.

were older and showed a significantly higher number of comorbidities as well as increased serum triglyceride levels (Table 3). Arterial stiffness measurements at baseline showed a trend for higher PWV values in patients who developed cardiovascular events during follow-up (Table 3).
Ten cardiovascular events occurred in 6 of the 31 patients (19.4\%) discontinuing PAP therapies. Seventeen cardiovascular events occurred in 13 of the 69 patients (18.8\%) who were still on treatment at the end of follow-up (CPAP: $n=54$; NIV: $n=15$ ). No significant difference in the nonfatal event rate was evident between the CPAP- and the 
Fig. 3. Cardiovascular event rate per year per 100 patients. Analysis by 2 -way analysis of variance (comorbidities: $p=0.097$, not significant; current PAP therapy: $p=$ 0.0095; interaction comorbidities $\times$ treatment: $p=0.0166)$. CPAP, continuous positive airway pressure; NIV, noninvasive ventilation; PAP, positive airway pressure.

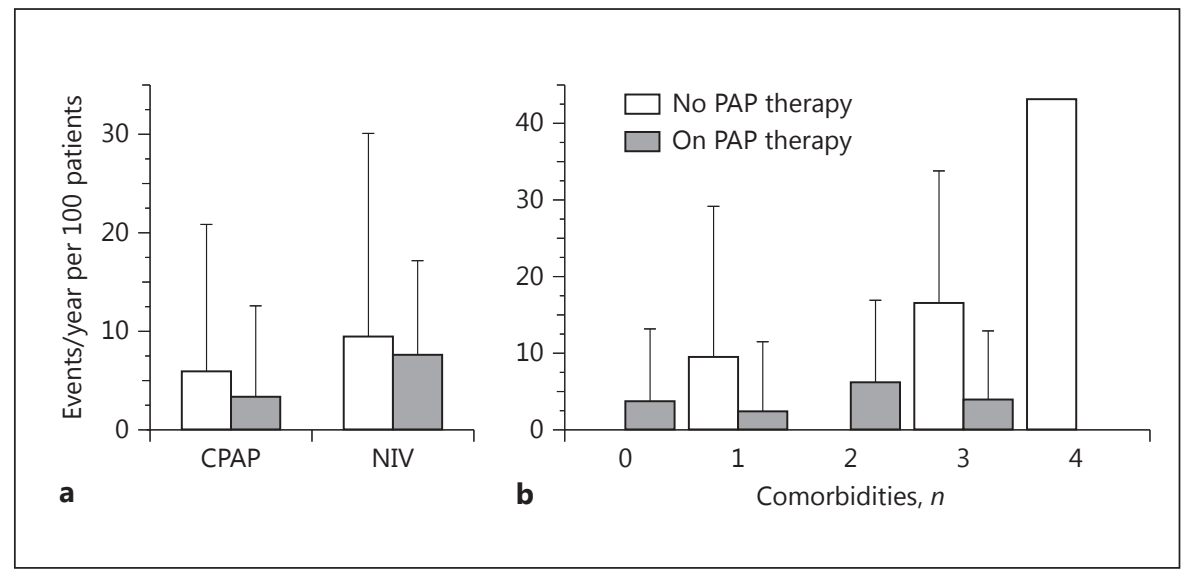

Fig. 4. Pharmacological treatment according to occurrence of cardiovascular events (white bars: without event; grey bars: with events). The $p$ values refer to the difference between patients without and with events $\left(\chi^{2}\right)$. ACE-I, angiotensin-converting enzyme I; ARB, angiotensin receptor blocker; ASA, acetylsalicylic acid; PPI, proton pump inhibitor.

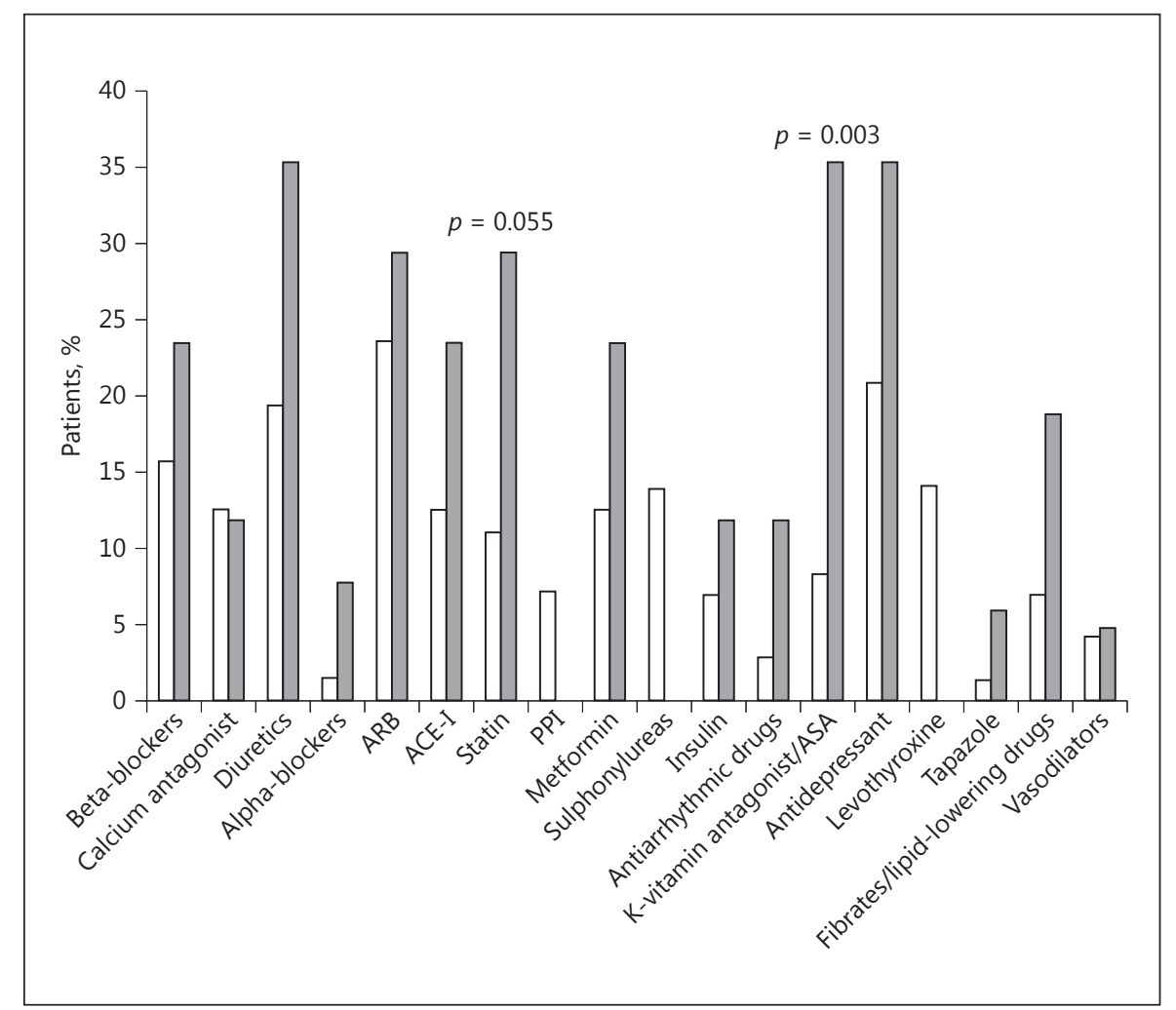

NIV-treated group (Fig. 3a). Two-way analysis of variance was used to analyze the effect of continuing/interrupted treatment, the impact of comorbidities, and their interaction in the entire group. In the entire cohort, the event rate was significantly lower in patients who were on PAP therapy compared to those who interrupted it $(p=0.0086)$. The event rate tended to increase with the number of comorbidities $(p=0.097)$, but there was a significant interaction between treatment and comorbidities $(p=0.017)$ (Fig. 3b).
During follow-up, 13 patients underwent bariatric surgery, 7 in the CPAP group (9.3\%) and 6 in the NIV group $(21.4 \%)(p=0.10)$. On average, these patients lost $12.5 \pm 8.9$ BMI units $(p<0.0001$ compared to the $0.1 \pm 5.1$ $\mathrm{BMI}$ unit change in the rest of the sample). The results of the analysis of the event rate were unchanged after exclusion of these 13 patients (current treatment: $p=0.0123$; comorbidities: $p=0.129$; interaction current treatment $\times$ comorbidities: $p=0.011$ ). 
Patients with events showed a significantly higher use of anticoagulant/antiplatelet drugs as well as a trend for more frequent statin and diuretic prescription in agreement with their high comorbidity burden (Fig. 4).

\section{Discussion}

In OSA patients with moderate to severe obesity, the majority of patients were highly compliant to nocturnal ventilatory treatment (over $6 \mathrm{~h} / \mathrm{night}$ in both the CPAP and the NIV group) and did not experience cardiovascular events. Late cardiovascular events mostly occurred in patients with multiple comorbidities who had interrupted PAP therapy. This suggests that long-term PAP therapies may effectively decrease cardiovascular risk and at least partly counteract the negative impact of both OSA and cardiometabolic comorbidities.

A major limitation of our study is the small sample size and the observational design. Patients with OHS were too few to perform a separate analysis; they were therefore excluded. There was no snoring control group, which would be very hard to select in patients with severe obesity. Also, data on cardiovascular events were derived from self-administered questionnaires and not prospectively assessed by an event committee. Finally, because the rate of interruption of treatment was higher among nonresponders than responders to the questionnaire, cardiovascular event rates might be somewhat underestimated by our study.

The originality and strength of our study are that highquality data regarding comorbidities and medications as well as data on surrogate markers of cardiovascular risk (arterial stiffness and endothelial function) were collected at diagnosis. This allowed us to identify a clinical and biological profile associated with late cardiovascular events.

The patients in our cohort were mostly treated with CPAP, with about one-fourth of the sample treated with NIV. In this cohort of OSA patients, NIV was mainly indicated in case of "CPAP failure"; however, we did not perform in-lab titration of CPAP, and the choice between CPAP and NIV was left at the discretion of the clinician in charge of the patient at the moment of initiation; this could have biased the interpretation of the comparison between the CPAP and the NIV group. Yet, the differences between treatment groups were small, and analysis of cardiovascular events was conducted in the whole sample. Although not significantly different, possibly because of the small sample size, the event rate tended to be higher in NIV- than in CPAP-treated patients, possibly be- cause they were in more severe overall clinical conditions, as suggested by the trend towards a higher prevalence of comorbidities in this group compared to patients on CPAP. Besides the expected effect of age, patients who developed events during follow-up showed a higher comorbidity burden and worse lipid profile. A recent large prospective cohort study demonstrated that OSA-related comorbidities are the most important predictors of composite cardiovascular outcomes [11], but provided no information about PAP adherence. Our study extends such results by showing that the impact of comorbidities is particularly significant in noncompliers to PAP treatment. Interruption of ventilatory treatment, however, could also be a marker of poor adherence to medications in general.

PWV is acknowledged as a strong predictor of late cardiovascular events [32]. PWV at baseline was on average higher in our patients compared to the available reference values adjusted for age and blood pressure [29]. It is conceivable that both obesity and sleep-disordered breathing might have contributed to increased PWV in our cohort, but the variables BMI, AHI, and nocturnal $\mathrm{SaO}_{2}$ did not enter the multiple regression model. Therefore, results in OSA patients with moderate to severe obesity differ from those obtained in OSA patients without cardiovascular comorbidities, in whom PWV increased with OSA severity $[33,34]$ and decreased after CPAP treatment [35]. In severely obese patients, CPAP treatment improved but did not normalize pulse wave analysis [36, 37]. Other positive short-term effects of CPAP on pulse wave analysis were recently reported by Korcarz et al. [38] in a younger and less obese population compared to our patients. In our cohort, patients with events during follow-up showed a trend for higher PWV at diagnosis, although the difference did not reach significance, probably owing to an insufficient sample size. A limitation of our study is the lack of longitudinal data on arterial stiffness.

Longitudinal analysis revealed a significant association of cardiovascular events with the comorbidity burden, but this was true only in patients who interrupted PAP therapies during follow-up (Fig. 3). Conversely, event rates were low in treated patients, even in the presence of multiple comorbidities. These data indicate a possible protective effect of PAP therapy, confirming and extending the results of previous studies.

The overall event rate per year in our cohort was 8 -fold higher (5.3 \pm 12.0 per 100 patients) than the nonfatal event rate of 0.64 events $/ 100$ patients per year reported by Marin et al. [8] in patients with severe OSA 
treated with CPAP, and over twice the event rate in patients with untreated severe OSA (2.13 events/100 patients per year) in the same cohort. Patients with cardiovascular events during follow-up were on more intense pharmacological treatment at diagnosis, but control of cardiovascular risk factors or lifestyle interventions during follow-up were not specifically assessed, indicating the opportunity for further studies in larger samples. However, no major change in BMI occurred in patients with or without cardiovascular events during follow-up, with the exception of the small group of patients who lost a considerable amount of weight after bariatric surgery. Our results underline the need to adopt a multimodal approach to correct the entire spectrum of cardiometabolic abnormalities beside correcting sleep-disordered breathing [17].

In conclusion, this longitudinal study in a cohort of moderately to severely obese OSA patients indicates a possible protective effect of long-term nocturnal ventilatory treatment on cardiovascular risk, especially in patients with a high comorbidity burden.

\section{Acknowledgments}

This project was funded by a European Respiratory Society Short-Term Research Fellowship (STRTF - 4008-2013) to Dr. Anna Maria Marotta, and grants from the Fond de dotation "Agir pour les maladies chroniques."

\section{Financial Disclosure and Conflicts of Interest}

J.-C. Borel is employed by AGIR à dom (a French nonprofit home care provider); he also received grants and personal fees from Philips Healthcare and nonfinancial support from RESMED. All the other authors declare no conflict of interest.

\section{Author Contributions}

A.M. Marotta, J.-C. Borel, J.L. Pépin, and M.R. Bonsignore conceived and designed the study. A.M. Marotta and L.M. Galerneau collected the data. A.M. Marotta and M.R. Bonsignore analyzed the data. A.M. Marotta, J.-C. Borel, R. Tamisier, J.L. Pépin, and M.R. Bonsignore wrote the paper. All of the authors approved the final draft of the manuscript.

\section{References}

1 Lavie CJ, De Schutter A, Milani RV: Healthy obese versus unhealthy lean: the obesity paradox. Nat Rev Endocrinol 2015;11:55-62.

2 Calle EE, Thun MJ, Petrelli JM, Rodriguez C, Heath CW Jr: Body-mass index and mortality in a prospective cohort of U.S. adults. N Engl J Med 1999;341:1097-1105.

3 Song X, Jousilahti P, Stehouwer CD, Söderberg S, Onat A, Laatikainen T, Yudkin JS, Dankner R, Morris R, Tuomilehto J, Qiao Q; DECODE Study Group: Cardiovascular and all-cause mortality in relation to various anthropometric measures of obesity in Europeans. Nutr Metab Cardiovasc Dis 2015;25:295304.

4 Kitahara CM, Flint AJ, Berrington de Gonzalez A, Bernstein L, Brotzman M, MacInnis RJ, Moore SC, Robien K, Rosenberg PS, Singh PN, Weiderpass E, Adami HO, Anton-Culver H, Ballard-Barbash R, Buring JE, Freedman DM, Fraser GE, Beane Freeman LE, Gapstur SM, Gaziano JM, Giles GG, Håkansson N, Hoppin JA, Hu FB, Koenig K, Linet MS, Park Y, Patel AV, Purdue MP, Schairer C, Sesso HD, Visvanathan K, White E, Wolk A, Zeleniuch-Jacquotte A, Hartge P: Association between class III obesity (BMI of $40-59 \mathrm{~kg} / \mathrm{m}^{2}$ ) and mortality: a pooled analysis of 20 prospective studies. PLoS Med 2014; 11:e1001673.

5 Salord N, Mayos M, Miralda R, Perez A: Respiratory sleep disturbances in patients undergoing gastric bypass surgery and their relation to metabolic syndrome. Obes Surg 2009;19: 74-79.

6 Gasa M, Salord N, Fortuna AM, Mayos M, Vilarrasa N, Dorca J, Montserrat JM, Bonsignore MR, Monasterio C: Obstructive sleep apnoea and metabolic impairment in severe obesity. Eur Respir J 2011;38:1089-1097.

7 Fox H, Purucker HC, Holzhacker I, Tebtmann U, Bitter T, Horstkotte D, Graml A, Woehrle H, Oldenburg O: Prevalence of sleep-disordered breathing and patient characteristics in a coronary artery disease cohort undergoing cardiovascular rehabilitation. J Cardiopulm Rehabil Prev 2016;36:421-429.

8 Marin JM, Carrizo SJ, Vicente E, Agusti AG: Long-term cardiovascular outcomes in men with obstructive sleep apnoea-hypopnoea with or without treatment with continuous positive airway pressure: an observational study. Lancet 2005;365:1046-1053.

9 Doherty LS, Kiely JL, Swan V, McNicholas WT: Long-term effects of nasal continuous positive airway pressure therapy on cardiovascular outcomes in sleep apnea syndrome. Chest 2005;127:2076-2084.

10 Kohler M, Ayers L, Pepperell JC, Packwood KL, Ferry B, Crosthwaite N, Craig S, Siccoli MM, Davies RJ, Stradling JR: Effects of continuous positive airway pressure on systemic inflammation in patients with moderate to severe obstructive sleep apnoea: a randomised controlled trial. Thorax 2009;64:67-73.
11 Kendzerska T, Gershon AS, Hawker G, Leung RS, Tomlinson G: Obstructive sleep apnea and risk of cardiovascular events and allcause mortality: a decade-long historical cohort study. PLoS Med 2014;11:e1001599.

12 Salord N, Gasa M, Mayos M, Fortuna-Gutierrez AM, Montserrat JM, Sánchez-de-la-Torre M, Barceló A, Barbé F, Vilarrasa N, Monasterio C: Impact of OSA on biological markers in morbid obesity and metabolic syndrome. J Clin Sleep Med 2014;10:263-270.

13 Kendzerska T, Leung RS, Gershon AS, Tomlinson G, Ayas N: The interaction of obesity and nocturnal hypoxemia on cardiovascular consequences in adults with suspected obstructive sleep apnea. A historical observational study. Ann Am Thorac Soc 2016; 13:2234-2241.

14 Hoyos CM, Killick R, Yee BJ, Phillips CL, Grunstein RR, Liu PY: Cardiometabolic changes after continuous positive airway pressure for obstructive sleep apnoea: a randomised sham-controlled study. Thorax 2012;67:1081-1089.

15 Pépin JL, Tamisier R, Lévy P: Obstructive sleep apnoea and metabolic syndrome: put CPAP efficacy in a more realistic perspective. Thorax 2012;67:1025-1027.

16 Budweiser S, Riedl SG, Jörres RA, Heinemann F, Pfeifer M: Mortality and prognostic factors in patients with obesity-hypoventilation syndrome undergoing noninvasive ventilation. J Intern Med 2007;261:375-383.
CPAP/NIV in Obese OSA Patients at

High Cardiovascular Risk 
17 Borel JC, Borel AL, Monneret D, Tamisier R, Levy P, Pepin JL: Obesity hypoventilation syndrome: from sleep-disordered breathing to systemic comorbidities and the need to offer combined treatment strategies. Respirology 2012;17:601-610.

18 Borel JC, Tamisier R, Gonzalez-Bermejo J, Baguet JP, Monneret D, Arnol N, Roux-Lombard P, Wuyam B, Levy P, Pépin JL: Noninvasive ventilation in mild obesity hypoventilation syndrome: a randomized controlled trial. Chest 2012;141:692-702

19 Priou P, Hamel JF, Person C, Meslier N, Racineux JL, Urban T, Gagnadoux F: Long-term outcome of noninvasive positive pressure ventilation for obesity hypoventilation syndrome. Chest 2010;138:84-90.

20 Borel JC, Burel B, Tamisier R, Dias-Domingos S, Baguet JP, Levy P, Pepin JL: Comorbidities and mortality in hypercapnic obese under domiciliary noninvasive ventilation. PLoS One 2013;8:e52006.

21 Borel JC, Pepin JL, Pison C, Vesin A, Gonzalez-Bermejo J, Court-Fortune I, Timsit JF: Long-term adherence with non-invasive ventilation improves prognosis in obese COPD patients. Respirology 2014;19:857-865.

22 Mancia G, Fagard R, Narkiewicz K, et al: 2013 ESH/ESC guidelines for the management of arterial hypertension: the Task Force for the Management of Arterial Hypertension of the European Society of Hypertension (ESH) and of the European Society of Cardiology (ESC). Eur Heart J 2013;34:2159-2219.

23 Pellegrino R, Viegi G, Brusasco V, Crapo RO, Burgos F, Casaburi R, Coates A, van der Grinten CP, Gustafsson P, Hankinson J, Jensen R, Johnson DC, MacIntyre N, McKay R, Miller MR, Navajas D, Pedersen OF, Wanger $\mathrm{J}$ : Interpretative strategies for lung function tests. Eur Respir J 2005;26:948-968.

24 Berry RB, Budhiraja R, Gottlieb DJ, Gozal D, Iber C, Kapur VK, Marcus CL, Mehra R, Parthasarathy S, Quan SF, Redline S, Strohl KP, Davidson Ward SL, Tangredi MM; American Academy of Sleep Medicine: Rules for scoring respiratory events in sleep: update of the 2007 AASM Manual for the Scoring of Sleep and
Associated Events. Deliberations of the Sleep Apnea Definitions Task Force of the American Academy of Sleep Medicine. J Clin Sleep Med 2012;8:597-619.

25 Johns MW: A new method for measuring daytime sleepiness: the Epworth sleepiness scale. Sleep 1991;14:540-545.

26 Matthews DR, Hosker JP, Rudenski AS, Naylor BA, Treacher DF, Turner RC: Homeostasis model assessment: insulin resistance and beta-cell function from fasting plasma glucose and insulin concentrations in man. Diabetologia 1985;28:412-419.

27 Alberti KG, Eckel RH, Grundy SM, Zimmet PZ, Cleeman JI, Donato KA, Fruchart JC, James WP, Loria CM, Smith SC Jr; International Diabetes Federation Task Force on Epidemiology and Prevention; National Heart, Lung, and Blood Institute; American Heart Association; World Heart Federation; International Atherosclerosis Society; International Association for the Study of Obesity: Harmonizing the metabolic syndrome: a joint interim statement of the International Diabetes Federation Task Force on Epidemiology and Prevention; National Heart, Lung, and Blood Institute; American Heart Association; World Heart Federation; International Atherosclerosis Society; and International Association for the Study of Obesity. Circulation 2009; 120:1640-1645.

28 Phillips CL, Butlin M, Wong KK, Avolio AP: Is obstructive sleep apnoea causally related to arterial stiffness? A critical review of experimental evidence. Sleep Med Rev 2012;17:718.

29 Boutouyrie P, Wermeersch SJ; Reference Values for Arterial Stiffness' Collaboration: Determinants of pulse wave velocity in healthy people and in the presence of cardiovascular risk factors: "establishing normal and reference values". Eur Heart J 2010;31: 2338-2350.

30 Nohria A, Gerhard-Herman M, Creager MA, Hurle S, Mitra D, Ganz P: Role of nitric oxide in regulation of digital pulse volume amplitude in humans. J Appl Physiol 2006;101: 545-548.
31 Huang AL, Silver AE, Shvenke E, Schopfer DW, Jahangir E, Titas MA, Shpilman A, Menzoian JO, Watkins MT, Raffetto JD, Gibbons G, Woodson J, Shaw PM, Dhadly M, Eberhardt RT, Keaney JF Jr, Gokce N, Vita JA: Predictive value of reactive hyperemia for cardiovascular events in patients with peripheral arterial disease undergoing vascular surgery. Arterioscler Thromb Vasc Biol 2007;27:2113-2119.

32 Vlachopoulos C, Aznaouridis K, Stefanadis C: Prediction of cardiovascular events and allcause mortality with arterial stiffness: a systematic review and meta-analysis. J Am Coll Cardiol 2010;55:1318-1327.

33 Drager LF, Bortolotto LA, Lorenzi MC, Figueiredo AC, Krieger EM, Lorenzi-Filho G: Early signs of atherosclerosis in obstructive sleep apnea. Am J Respir Crit Care Med 2005; 172:613-618

34 Pépin JL, Tamisier R, Baguet JP, Lévy P: Arterial health is related to obstructive sleep apnea severity and improves with CPAP treatment. Sleep Med Rev 2013;17:3-5.

35 Drager LF, Bortolotto LA, Figueiredo AC, Krieger EM, Lorenzi-Filho GF: Effects of continuous positive airway pressure on early signs of atherosclerosis in obstructive sleep apnea. Am J Respir Crit Care Med 2007;176: 706-712.

36 Seetho IW, Asher R, Parker RJ, Craig S, Duffy N, Hardy KJ, Wilding JP: Effect of CPAP on arterial stiffness in severely obese patients with obstructive sleep apnoea. Sleep Breath 2015;19:1155-1165.

37 Mineiro MA, Marques da Silva P, Alves M, Virella D, Marques Gomes MJ, Cardoso J: Use of CPAP to reduce arterial stiffness in moderate-to-severe obstructive sleep apnoea, without excessive daytime sleepiness (STIFFSLEEP): an observational cohort study protocol. BMJ Open 2016;6:e011385.

38 Korcarz CE, Benca R, Barnet JH, Stein JH: Treatment of obstructive sleep apnea in young and middle-aged adults: effects of positive airway pressure and compliance on arterial stiffness, endothelial function, and cardiac hemodynamics. J Am Heart Assoc 2016; 5:e002930. 